\title{
Deep Learning Framework to Detect the False Analysis of a Product given by Robots and Malicious Users
}

\author{
Sunil Bhutada, V.V.S.S.S. Balaram, Challa Akshara Sree
}

\begin{abstract}
Product evaluations are precious for upcoming clients in supporting them make choices. To this, numerous mining techniques have been proposed, wherein judging a evaluation sentence's orientation (e.g. Outstanding or bad) is considered as one of their key worrying conditions. Lately, deep studying has emerged as a powerful technique for fixing sentiment kind issues. A neural network intrinsically learns useful instance routinely without human efforts. But, the fulfilment of deep getting to know pretty is primarily based totally on the supply of big-scale education data. We recommend a unique deep studying framework for product review sentiment classification which employs prevalently to be had rankings as susceptible supervision signs and symptoms. The framework consists of steps: (1) studying a high level representation (an embedding region) which captures the general sentiment distribution of sentences thru score facts; (2) such as a class layer-on top of the embedding layer and use labelled sentences for supervised fine-tuning. We discover styles of low stage community structure for modelling evaluation sentences, specifically, convolution function extractors and prolonged brieftime period memory. To have a take a look at the proposed framework, we gather a data set containing 1.1M weakly classified evaluate sentences and eleven, 754 labelled review sentences from Amazon. Experimental effects display the efficacy of the proposed framework and its superiority over baselines. In this future work todetect false reviews given by robots or by malicious people by taking amount, sometimessome companies may hire people to boost their product ranking higher by assigning fake rating and this malicious people or robots give continuous ranking or review to such product and we can detect such fake rating by analysingratingandremove suchfake rating to give only genuine reviews to users.
\end{abstract}

Keywords:-Sentiment Classification, Weak Supervision, Feature Extractors, Deep learning.

\section{INTRODUCTION}

With the booming of e-trade, human beings have become used to consuming on line and writing remarks approximately their buy reviews on merchant/evaluations internet web sites. Those opinionated contents are precious belongings every to future customers for desire-making and to traders for boosting their products and/or service. However, due to the reality the volume of critiques grows swiftly, people being ought to face a severe statistics

Revised Manuscript Received on April 12, 2019.

Sunil Bhutada,Professor, IT Department Sreenidhi Institute of Science \& Technology Yamnampet, Ghatkesar, Hyderabad. Telangana, India. (sunilb@sreenidhi.edu.in)

V.V.S.S.S. Balaram,Professor,Head of IT Department Sreenidhi Institute of

Science

\&TechnologyYamnampet,Ghatkesar,Hyderabad.Telangana,

India.(vbalaram@sreenidhi.edu.in)

ChallaAksharaSree, M.Tech II year , IT Department Sreenidhi Institute of Science \&Technology Yamnampet,Ghatkesar,Hyderabad. Telangana, India.(akshara.challa3@gmail.com)

overload hassle. To relieve this problem, many opinion mining strategies have been proposed, e.g. Opinion summarization opinion polling, and comparative evaluation. The important thing mission is a manner to accurately assume the sentiment orientation of evaluation sentences.

Famous sentiment category techniques generally fall into instructions: (1) lexicon-primarily based strategies and (2) Machine learningstrategies. Lexicon based completely strategies normally take the tack of first building a sentiment lexicon of opinion terms(e.g. "extremely good", "disgusting"), after which layout category regulations based totally on seemed opinion terms and in advance syntactic facts. Regardless of effectiveness, this shape of techniques requires massive efforts in lexicon creation and rule format. Furthermore, lexicon-based totally techniques cannot nicely deal with implicit evaluations, i.e. Intention statements collectively with "I bought the mattress in step with week within the beyond, and a valley appeared nowadays". As cited in, that is furthermore an important form of reviews. Real records is usually greater beneficial than subjective feelings. Lexicon-based strategies can maximum effective deal with implicit evaluations in an advert-hoc way.

The first machine learning based sentiment classification work applied popular machine learning algorithms which includes Naïve Bayes to the problem. After that, most research in this course revolved around feature engineering for higher classificationperformance. Different styles of features were explored, e.g. N-grams, part-of-speech (POS) records and syntactic relations and plenty of others. Feature engineering additionally costs of several human efforts, and a feature set suitable for one area won't generate precise average overall performance for other domains.

In modern day years, deep studying has emerged as a powerful way for fixing sentiment class problems. A deep neural network intrinsically learns a high degree representation of the records, for this reason preserving off hard works including function engineering. A second advantage is that deep models have exponentially stronger expressive electricity than shallow strategies. However, the achievement of deep mastering carefully is predicated at the delivery of massive-scale training statistics. Labelling a Huge form of sentences may be very hard.

Fortunately, maximum provider issuer/review websites allow clients to summarize their opinions through an everyday score rating (usually in 5-stars scale). Ratings reflect the general sentiment of client opinions and characteristic already been exploited for sentiment

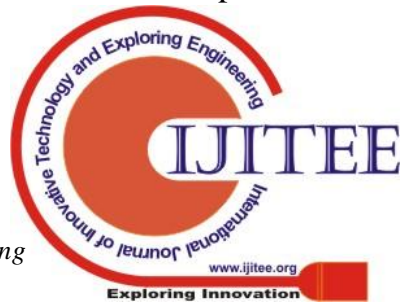


evaluation. Nonetheless, assessment ratings aren't dependable labels for the constituent sentences, e.g. A fivestars observe can encompass horrible sentences and we may also moreover see first-rate phrases once in a while in 1celebrity opinions.

An instance is confirmed. Consequently, treating binaries rankings as sentiment labels may also moreover need to confuse a sentiment classifier for evaluation sentences. No depend the promising not unusual well-known universal performance of deep gaining knowledge of on sentiment category, no preceding paintings tried to leverage the prevalently to be had rankings for schooling deep models. In this framework, we suggest a unique deep learning framework for evaluation sentence sentiment class. The framework treats evaluation ratings as susceptible labels to teach deep neural networks. As an example, with 5-movie star scale we're able to deem rankings above/beneath threestars as great/ horrific susceptible labels respectively. The framework typically includes steps. Inside the first step, instead of predicting sentiment labels right now, we attempt to research and embedding location (a high stage layer in the neural community) which displays the huge sentiment distribution of sentences, from a big amount of weakly labelled sentences. This is, we pressure sentences with the identical susceptible labels to be close to every one-of-atype, on the equal time as sentences with particular susceptible labels are saved a long way from each unique. To reduce the effect of sentences with score-inconsistent orientation (hereafter known as incorrect-categorized sentences), we advise to penalize the relative distances among sentences in the embedding space thru a rating loss. Inside the $2 \mathrm{~d}$ one step, a class layer is added on top of the embedding layer, and we use classified sentences to greatmusic the deep network. The framework is dubbed Weaklysupervised Deep Embedding (WDE). Regarding network form, two well-known schemes are observed to discover ways to extract consistent-length characteristic vectors from observe sentences, mainly, convolution characteristic extractors and lengthy brief term memory (LSTM). With a moderate abuse of idea, we are able to seek advice from the previous model as Convolution Neural community primarily based WDE (WDE-CNN); the latter one is called LSTM based totally WDE (WDE-LSTM). We then compute high diploma capabilities (embedding) thru synthesizing the extracted capabilities, similarly to the contextual detail statistics (e.g. Screen of cell phones) of the product. The hassle enters represents preceding data regarding the sentence's orientation.

The essential contributions of this paper are summarized as follows: 1) we propose a new deep mastering framework WDE that can leverage the big quantity of weakly categorized review sentences for sentiment evaluation. The framework first attempts to seize the sentiment distribution of the facts through embedding training on weakly classified sentences. Then it uses a few labelled sentences for deep community great tuning, further to for prediction version getting to know. We empirically display this "weakly preeducation + supervised first-class-tuning" idea is feasible. The concept could also be beneficial for exploiting special

sorts of weakly classified data (e.g. Tagging facts). 2) We devise preferred neural network architecture for WDE and instantiate it via using two famous neural community schemes for modelling textual content statistics: CNN and LSTM. We examine WDE-CNN and WDE-LSTM in phrases of their effectiveness, performance and specialties on this sentiment beauty undertaking. 3) To take a look at WDE we assemble a dataset containing $1.1 \mathrm{M}$ weakly labelled evaluation sentences and eleven,754 categorized evaluation sentences from three domain names of Amazon, i.e. Digital cameras, cell phones and laptops. The dataset can be downloaded. The rest of the paper is prepared as follows: the following segment outlines associated artwork. Sections are gives the WDE framework and explains the layout of every step in details.

\section{METHODOLOGY}

\section{A. WDE-CNN:}

The network structure of WDE-CNN, depicted, is a version of the CNNs described. In what follows, we use pinnacle case formidable letters in conjunction with $\mathrm{W}$ to signify matrices and lower case bold letters which embody $\mathrm{x}$ to indicate column vectors. The $\mathrm{i}$-th element in vector $\mathrm{x}$ is denoted via $\mathrm{x}(\mathrm{i})$.

\section{Input Layer:}

An enter sentence of length $t$ is a word collections $=\left\langle w_{1}, w_{2} \ldots w_{T}\right\rangle$. Every word $\omega$ within the vocabulary is defined via a phrase vector $\mathrm{x}$. Allow $\mathrm{k}$ be the length of $\mathrm{x}$ and $\mathrm{n}$ be the complete kind of phrases inside the vocabulary. The trainable phrase studies table $X$ is then a $\mathrm{k} \times \mathrm{n}$ matrix with phrase vectors as itscolumns. The input layer absolutely mapss $=\left\langle w_{1}, w_{2} \ldots . w_{T}\right\rangle$ toits corresponding phrase vector example $\left\langle X_{1}, X_{2} \ldots X_{T}\right\rangle$. The studies desk is initialized the use of the publicly to be had three hundreddimensional word vectors knowledgeable on hundred billion terms fromGoogle data via using way of word2vec. Out-ofsample terms are initializedrandomly.

\section{Convolutional Layer and Max pooling Layer.}

The convolutional layer applies a set of filters at the sentence. Each clear out $\mathrm{w} \in \mathbb{R}^{h k}$ is completed to a window of $h$ phrases to offer a nearby feature value:

$$
u(t)=f\left(\mathbf{w}^{T} \mathbf{x}_{t:(t+h-1)}+b\right)
$$

The max pooling layer plays a max operationovereach characteristic map $u_{j}$ to discover the maximum salient charge of the filter's corresponding characteristic as its final value

$$
v(j)=\max _{t}\left\{u_{j}(t)\right\}
$$

The max pooling layer performs a max operation over each feature map $u_{j}$ to discover the most salient fee of the filters corresponding characteristic as its last rate. 


\section{HIDDEN LAYER AND EMBEDDING LAYER:}

The constant-duration characteristic vector $\mathrm{v}$ is then fed to the sincerely associated hidden layer and embedding layer to extract nonlinear higher stage features. For the hidden layer, the computation is easy with weigh matrix $W_{h}$ and bias vector $b_{h}$ :

$$
\mathbf{h}=f\left(\mathbf{W}_{h} \mathbf{v}+\mathbf{b}_{h}\right)
$$

The embedding layer receives it's enter from assets: the output of the hidden layer $\mathrm{h}$, and context vector $a_{s}$ of sentence s. Context vectors of all elements constitute the context studies table A (as columns). The embedding layer output is computed as

$$
\mathbf{y}=f\left(\mathbf{W}_{e}\left[\begin{array}{l}
\mathbf{h} \\
\mathbf{a}_{s}
\end{array}\right]+\mathbf{b}_{e}\right)
$$

\section{CLASSIFICATIONLAYER:}

This layer is in fully connected to the embedding layer and outputs sentiment prediction for the enter sentence. It isn't always till the supervised pinnacle high-quality-tuning section that the layer is probably delivered to the community. We defer the outline of this residue.

\section{B. WDE-LSTM:}

The convolutional filters in WDE-CNN can handiest capture inputpatterns from a textual content window, e.g., of three phrases. It is tough for a CNNversion to explicitly capturelengthy-time period dependencies in sequentialinformation. Therefore, we propose each different instantiation of WDEwith the aid of lengthyShort-time period memory (LSTM). LSTM is a well-known methodfor recurrent neural networks (RNNs). An RNN step by means of stepupdates its state given the modern-day input and the previoushidden state from the final time step, making it a herbal preference formodelling sequential records which encompass herbal language sentences.LSTM has nowadays attracted hundreds interest due to its capacity to examine lengthy-time period dependencies through using a gating mechanism.

A LSTM keeps a memory cell that might betaken into attention as a non-save you analogy of a memory circuit. The reminiscence cellular controls the test, write and reset operations of its internal nation via output, input and neglect about gates respectively, permitting the gradient data to be once more propagated via many time steps. Theforwardcomputation of a block of reminiscence cells at timet.

$$
\begin{aligned}
\mathbf{d}_{t} & =g\left(\mathbf{W}_{\mathbf{d}} \mathbf{x}_{t}+\mathbf{U}_{\mathrm{d}_{t-1}} \mathbf{z}_{t} \mathbf{b}_{\mathbf{d}}\right) \\
\mathbf{i}_{t} & =\sigma\left(\mathbf{W}_{\mathbf{i}} \mathbf{x}_{t}+\mathbf{U}_{\mathbf{i}} \mathbf{z}_{t-1}+\mathbf{b}_{\mathbf{i}}\right) \\
\mathbf{f}_{t} & =\sigma\left(\mathbf{W}_{\mathrm{f}} \mathbf{x}_{t}+\mathbf{U}_{\mathrm{f}} \mathbf{z}_{t-1}+\mathbf{b}_{\mathbf{f}}\right) \\
\mathbf{o}_{t} & =\sigma\left(\mathbf{W}_{\mathbf{o}} \mathbf{x}_{t}+\mathbf{U}_{\mathbf{o}} \mathbf{z}_{t-1}+\mathbf{b}_{\mathbf{o}}\right) \\
\mathbf{c}_{t} & =\mathbf{i}_{t} \odot \mathbf{d}_{t}+\mathbf{f}_{t} \odot \mathbf{c}_{t-1} \\
\mathbf{z}_{t} & =\mathbf{o}_{t} \odot g\left(\mathbf{c}_{t}\right)
\end{aligned}
$$

A bidirectional shape generates a greater comprehensive character illustration of every phrase by manner of encoding contextual statistics from the whole sentence. At the opportunity hand, memory cells in LSTMs can be appeared as function extractors for detecting sequential patterns in sentences. Consequently, we again carry out max pooling over the whole phrase collection of a sentence to reap the most salientvalueof every extractor, forming the regularlength characteristic vector v: The layers above max pooling layer is similar to in WDE-CNN.

\section{RESULTS DISCUSSIONAND COMPARISION}

Enter the node size (the number of nodes to be created into the network) and select the node energy (the energy assigned for each node initially).

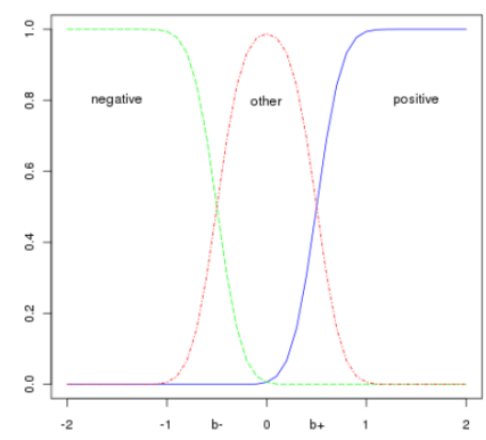

In existing system the above graph is for positive and negative reviews are displayed.

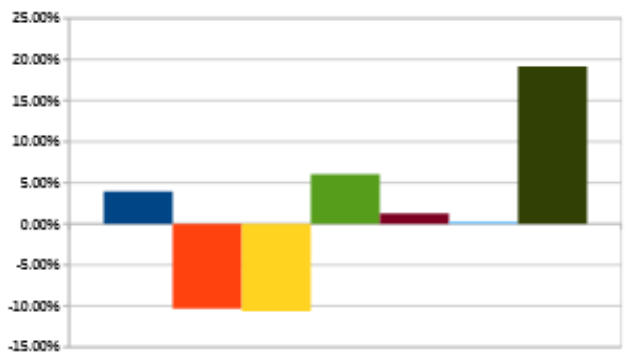

(c) LSTM

In existing system the positive and negative reviews are measured in the graph by LSTM method.

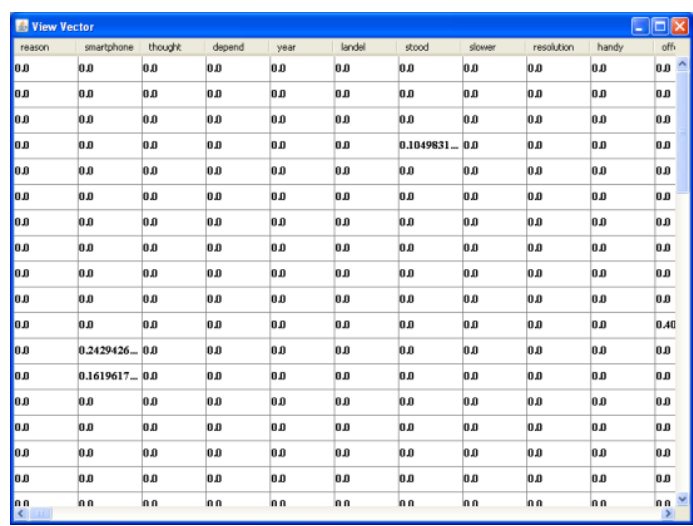


The window shows the extracted features in our proposed framework.

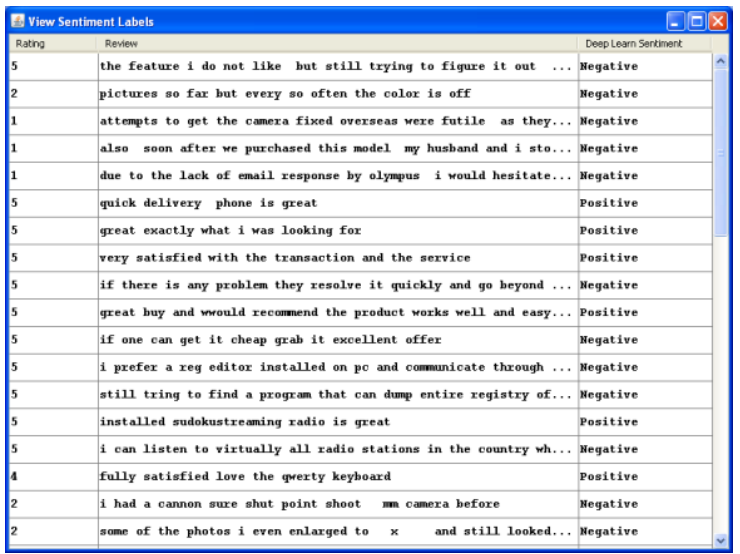

From above screen in first row rating is 5 but review text contains negative words and later by using this algorithm we found out that review is negative. Now click on 'Sentiment Prediction Graph' button to give input as review and then this input will test again train model to detect weather input is positive or negative.

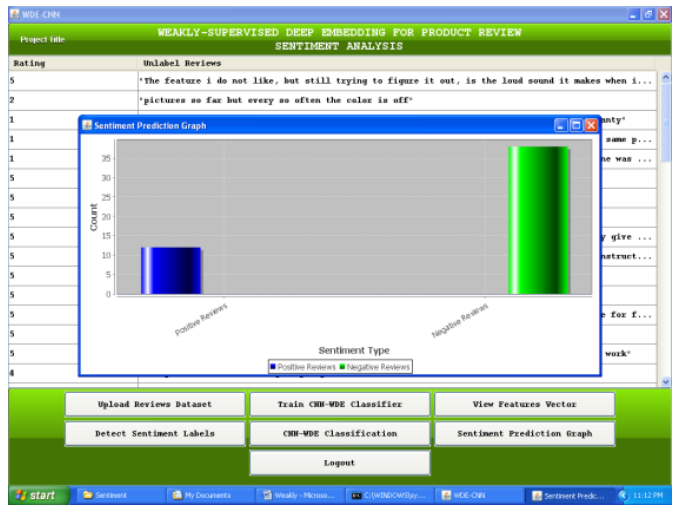

In proposed system the above graph is for negative and positive reviews.

\section{CONCLUSION}

In this work, we proposed a novel deep getting to know framework named Weakly-supervised Deep Embedding for have a look at sentence sentiment kind. WDE trains deep neural networks thru exploiting rating statistics of critiques it really is prevalently to be had on many service company/evaluate websites. The education is a 2-step approach: first we've got a take a look at an embedding region which tries to capture the sentiment distribution of sentences through penalizing relative distances among sentences consistent with susceptible labels inferred from rankings; then a softmax classifier is delivered on top of the embedding layer and we brilliant-tune the community through way of categorized statistics. Experiments on critiques accumulated

From Amazon.Com show that WDE is robust and outperforms baseline strategies. Unique instantiations of the framework, WDE-CNN and WDE-LSTM, are proposed. In contrast to WDE-LSTM, WDE-CNN has fewer model parameters, and its computation is without trouble parallelized on GPUs. However, WDE-CNN can't well cope with long-term dependencies in sentences.

WDE-LSTM is greater able to modelling the extendedterm dependencies in sentences; but it is lots an entire lot much less green than WDE-CNN and goals extra training data. For destiny art work, Todiscover false critiques given with the aid of robots or with the aid of malicious peoples by way of taking amount, sometime some groups may additionally hire humans to boost their product and their product rating better by using assigning fake rating and this malicious peoples or robots give continuous ranking or assessment to such product and we will come across such faux score with the aid of studying score and do away with such faux score to give only genuine proper opinions to customers.

\section{REFERENCES}

1. Y. Bengio. Learning deep architectures for ai. Foundations and trends in Machine Learning, 2(1):1-127, 2009.

2. L. Qu, R. Gemullaand G. Weikum. A weaklysupervisedmodel for sentence-level semantic orientation analysis with multiple experts. In EMNLP-CoNLL, pages 149-159, 2012.

3. C. M. Bishop. Pattern recognition and machine Learning.Springer, 2006.

4. X. Ding, B. Liu, and P. S. Yu.A holistic lexicon-based approach to opinion mining. In WSDM, pages 231-240, 2008.

5. Y. Kim. Convolutional neural networks for sentence classification.In EMNLP, pages 1746-1751, 2014.

6. H. Halpin, V. Robu, and H. Shepherd.The complex dynamics of collaborative tagging. In WWW, pages 211-220, 2007.

7. S. Hochreiter and J. Schmidhuber.Long short-term memory. Neural computation, 9(8):1735-1780, 1997.

8. M. Hu and B. Liu. Mining and summarizing customer reviews. In SIGKDD, pages 168-177, 2004. 